\title{
Cubic Atom and Crystal Structures
}

\author{
Zhiliang Cao ${ }^{1,2, *}$, Henry Gu Cao ${ }^{3}$ \\ ${ }^{1}$ Wayne State University, $42 \mathrm{~W}$ Warren Ave, Detroit \\ ${ }^{2}$ Shanghai Jiaotong University, Shanghai, China \\ ${ }^{3}$ Northwestern University, 633 Clark St, Evanston, IL 60208 \\ *Corresponding author: williamcao12252000@yahoo.com
}

Received September 12, 2014; Revised December 01, 2014; Accepted December 15, 2014

\begin{abstract}
The paper "Unified field theory" (UFT) unified four fundamental forces with help of the Torque model. UFT gives a new definition of Physics: "A natural science that involves the study of motion of space-time-energyforce to explain and predict the motion, interaction and configuration of matter." One of important pieces of matter is the atom. Unfortunately, the configuration of an atom cannot be visually observed. Two of the important accepted theories are the Pauli Exclusion Principle and the Schrodinger equations. In these two theories, the electron configuration is studied. Contrary to the top down approach, UFT theory starts from structure of Proton and Neutron using bottom up approach instead. Interestingly, electron orbits, electron binding energy, Madelung Rules, Zeeman splitting and crystal structure of the metals, are associated with proton's octahedron shape and three nuclear structural axes. An element will be chemically stable if the outmost s and p orbits have eight electrons which make atom a symmetrical cubic. Most importantly, the predictions of atomic configurations in this paper can be validated by characteristics of chemical elements which make the UFT claims credible. UFT comes a long way from spacetime-energy-force to the atom. The conclusions of UFT are more precise and clearer than the existing theories that have no proper explanation regarding many rules, such as eight outer electrons make element chemically stable and the exception on Madelung's rules. Regardless of the imperfections of the existing atomic theories, many particle Physics theories have no choice but to build on top of atomic theories, mainly Pauli Exclusion Principle and Schrodinger equations. Physics starts to look for answer via ambiguous mathematical equations as the proper clues are missing. Physics issues are different from mathematical issues, as they are Physical. Pauli Exclusion works well in electron configuration under specific physical condition and it is not a general Physics principal. Schrodinger's mathematical equations are interpreted differently in UFT. UFT is more physical as it built itself mainly on concept of Space, Time, Energy and Force, in the other word, UFT is Physics itself. Theory of Everything (ToE), the final theory of the Physics, can be simply another name for UFT. This paper connects an additional dot to draw UFT closer to ToE.
\end{abstract}

Keywords: nuclear physics, particle physics, unified field theory, gold, crystal

Cite This Article: Zhiliang Cao, and Henry Gu Cao, "Cubic Atom and Crystal Structures.” International Journal of Physics, vol. 2, no. 6 (2014): 277-281. doi: 10.12691/ijp-2-6-11.

\section{Introduction}

Unified Field Theory (UFT) [1-8] predicts that a proton has an octahedron [1] shape. The positive electronic charge forces from nuclei are vertical to the faces of the octahedron. The electrons of an atom are interacting with the tri-axes of the protons. Contrary to the common consensus that an electron in atom has a circular orbit, UFT predicts that the electron collides against a face of a proton in the nucleus where electronic field is strong. A proton face make an electron stay on the face itself for stability reasons.

Evenly allocated eight electrons vertical to the octahedron proton surface are points (vertices) of a cube. The wave characteristics of the electron decide the shell of the electron to maintain wave resonance. The wave interactions with the three axes of a proton split a point of cube into three triangle points on the second shell. In general, the cubic points are split into triangle grids' points.
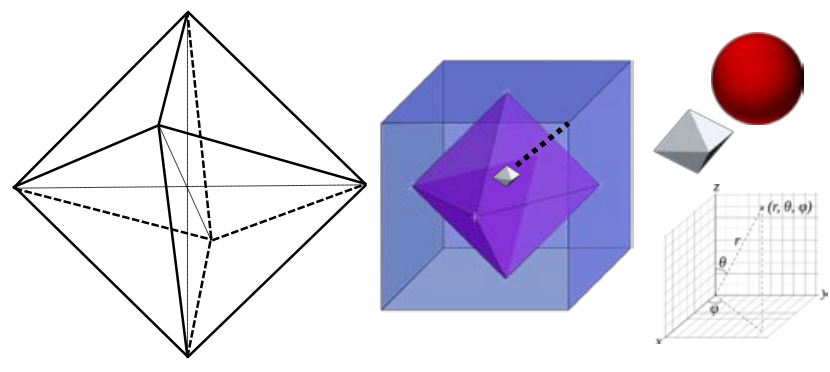

Figure 1. Octahedron Proton and Electron Movements

A possible electron configuration is result of resonance of electronic mass waves and nuclear internal waves. The ground state electron configuration for an element has maximum resonance. Noticeably, the crystal structures of the metals are related to the electron configuration and their orbit angle to the vertical line of the octahedron faces. 


\section{Results}

\subsection{Electron Movements}

Since the nuclei are octahedron shape [1,2], the electronic primary movement direction is vertical to the eight faces of the octahedron. In the other word, an electron collides with one of eight faces of proton and bounces like a ball. Even though an electron has a steady straight line "orbit", mass waves create wave patterns as they interact with three axes. The "orbit" of electron is from nucleus to a fixed high possibility point of wave pattern.

When the atomic number is one, the nuclei of hydrogen isotopes are shaped as dot or line. The movement of electron makes the nucleus constantly spinning in the gas state in normal temperature. Helium isotopes have line shaped nucleus. When the atomic number (proton count) is greater than two, the nuclei become 2D symmetrical plate or 3D octahedron piles.

The topology of nuclei provides an important clue to reexam the existing theories [9-41].

\subsection{Cubic Atom}

When each octahedron face has an outer shell electron vertical to the face, the outer shell electrons form a cubic for a noble gas elements with eight out most s/p orbits (except helium).

\subsection{Triangle Grids and Electron Orbits}

The three axes of nucleus interact with the electron mass waves and create diffraction pattern that is similar to the crystalline diffraction. The charged axis has one electronic field interaction, the two uncharged axes have two charged interactions each, positive and negative. Therefore, the charged axis has one wave and uncharged axes have two waves each. The proton/electron diffraction pattern is a triangle pattern. Various electron orbits are related to the layers of triangle strips. Each triangle node represents an "orbit". Each node is mirrored with a node on the opposite side of proton face.

$$
\text { p: } 3 * 2, d: 5 * 2, f: 7 * 2
$$
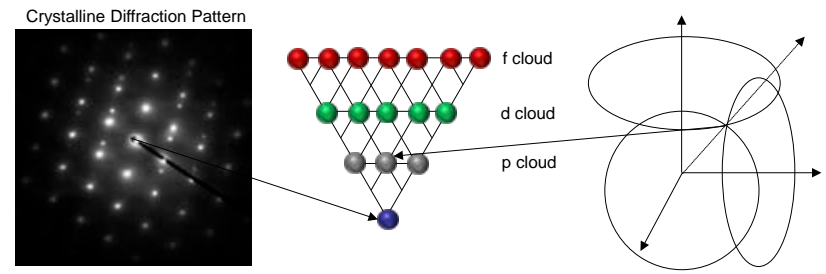

Figure 2. Electron Diffraction Triangle Grids and Zeeman Splitting

Electrons are evenly distributed vertical to eight octahedron faces. The possibility of the various electron orbits are based on the strength of electron's mass wave. The orbit s has highest priority for its high possibilities. The priority order is:

$\mathrm{s}, \mathrm{p}, \mathrm{d}$ and $\mathrm{f}$

In many cases, due to the shape of nucleus, there are exceptions to Madelung rules.

\subsection{Orbit Allocations}

To simplify the case, the wave function of the orbit on the shell is:

$$
\begin{aligned}
& \mathrm{Wx}+\mathrm{Wy}+\mathrm{Wz} \\
& \mathrm{Wx}=(2 / 3) \mathrm{Pi} \sin (16 \mathrm{~N} \mathrm{Ax}) \\
& \mathrm{Wy}=(2 / 3) \mathrm{Pi} \sin (16 \mathrm{~N} \mathrm{Ay}) \\
& \mathrm{Wz}=(2 / 3) \mathrm{Pi} \sin (8 \mathrm{~N} \mathrm{Az})
\end{aligned}
$$

$\mathrm{N}$ : shell count

Ax: Angle relative to $\mathrm{X}$

Ay: Angle relative to $\mathrm{Y}$

Az: Angle relative to $\mathrm{Z}$ (charged axis)

These three waves are independent waves.

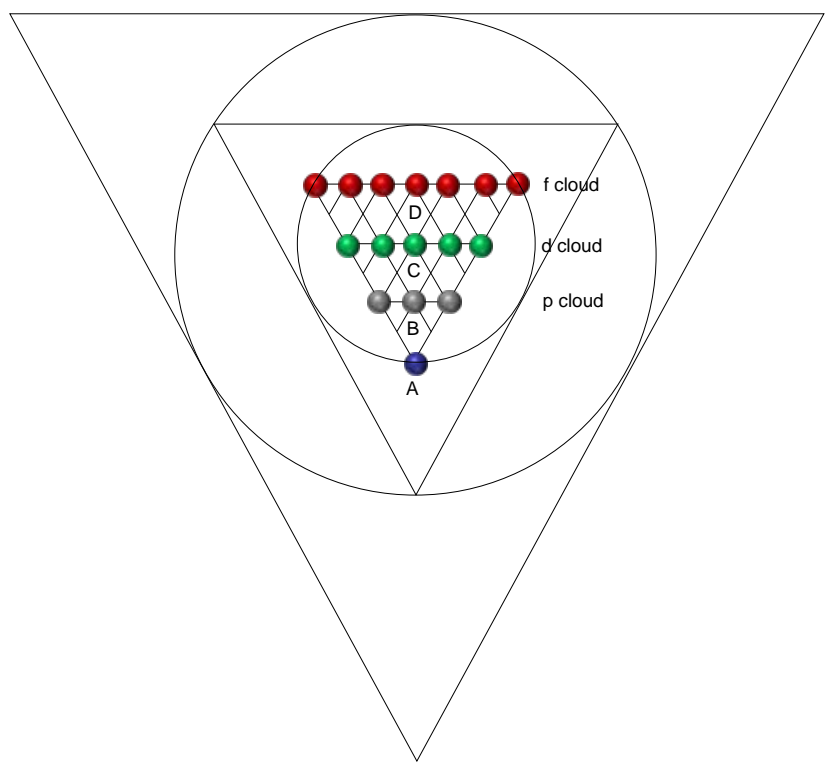

Figure 3. Triangle Grids Location

Even though the electron field vector is from the center of the triangle due to the resonance of the forces, the $\mathrm{S}$ orbit is closer to the charged axis.

\subsection{Multi-Atom Resonance}

When two atoms are close enough, the overlapped shells will have wave resonance as electrons are shared between the two atoms. The wave resonance not only provides straight orbits for the shared electrons, but also merges overlapped triangle grids from two neighboring atoms.

As result the new wave function is:

$\mathrm{W} 1+\mathrm{W} 2$

When different chemical atoms are mixed together, the molecule structure and wave resonance are related.

\section{Discussion}

\subsection{Quantum Tunnel Effects}

As electrons collide with nucleus, the collisions between outer electrons and inner electrons are unavoidable. These collisions are one of reasons for tunneling effect of inner electrons to get out of their orbits.

\subsection{Orbit Overlaps}

Since the mass waves do not stop at the nodes, the orbit of S, P, D and F extend themselves. The orbit extensions of the neighboring atoms overlap themselves. 


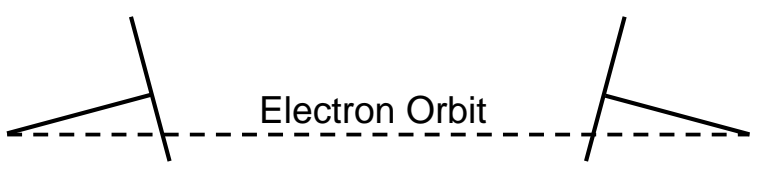

\section{Grids' Extension overlap}

Figure 4. Grids overlap

The above model tells us how tilted axes give a straight orbit for a shared electron.

The orbits are distorted by the nuclear forces, but they are mostly straight and symmetrical to have better resonance.

\subsection{Multiple Shells' Overlaps}

The D orbits' overlaps coexist with the S orbit and inner orbit overlap. For the element of the same period in element periodic table, the electron count is reverse proportional to the distance of the neighboring atom, as the overlapping increases the stabilities.

Propensities as follow reverse proportional to the wave strength of the orbits:

$$
\mathrm{n}+(1.6233 / 2) *(1+1)
$$

Wave interactions need the similar propensity values to make interaction waves with better contrasts. Most of interactions of the same elements neighboring to one another are the orbits with same quantum numbers, mainly the ones with high propensities. As the waves of these orbits can impact the configuration of electrons and the electron configuration is less stable.

\subsection{Crystal Structures of Beryllium}

Beryllium has a pair of S orbit with angle of A against the line vertical to an octahedron face. It makes Beryllium Proton axes not perfectly line up with the neighboring atoms even with higher temperature. Beryllium has HCP structure under the room temperature.
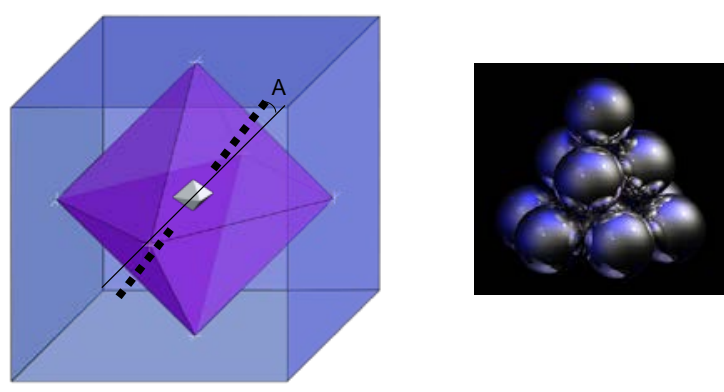

Figure 5. Beryllium HCP Crystal

\subsection{Crystal Structures of Calcium}

Calcium has a pair of S orbit. It makes Calcium's Proton axes not perfectly line up with the neighboring atoms. Unlike BCC Potassium, Calcium has FCC structure under the room temperature.

\subsection{Crystal Structures of Lithium}

The crystal structure of Lithium [42-51] near 0K is hR9. The hR9 crystal is largely due to the S orbit of the outmost shell is not at the center of the orbital triangle grids. At the room temperature, the rotation movement of nuclei allows nuclei "catch" the off central single S orbit electron when the structure [52-61] is bcc.

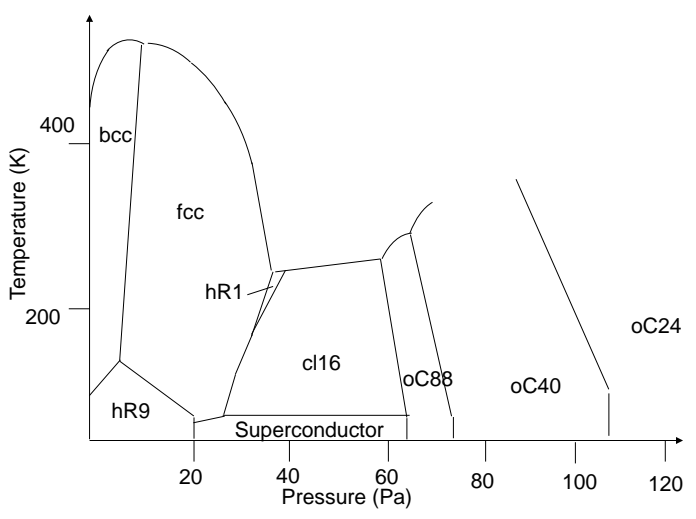

Figure 6. Lithium Phase Diagram

The existing crystallization theories [62-70] provide explanations for the crystal preferences under the pressure mainly based on entropy. The topology of atom provides visual explanations. Under the pressure of 10 to $40 \mathrm{~Pa}$, inner shell $\mathrm{S}$ orbit electrons become main factors. FCC crystal structure is the result of inner $1 \mathrm{~S}$ electron interactions. Since the $1 \mathrm{~S}$ electron has angle A, it prefers FCC structure which does not have perfectly lined up octahedron faces.

When the pressure reaches to $30 \mathrm{~Pa}$, the structure becomes complex cl16 and each octahedron face bind with two neighboring atoms. Lithium phase diagram is complex, but its complexities are topology of octahedron nuclei.

\section{Methods}

\subsection{S Orbit Angle and Phase}

$\mathrm{S}$ orbit is tilted an angle ("A" in Figure 5). As proton count increases, the angle is decreasing as the more protons join the interaction processes to bring $\mathrm{S}$ orbit to the center:

Be hcp

Mg hcp

Ca fcc

Sr fcc

Ba bcc

Ra bcc

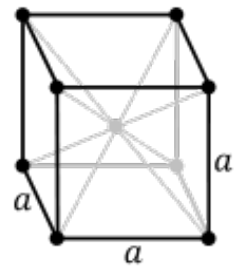

bcc fcc

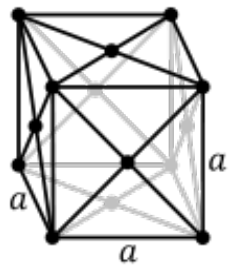

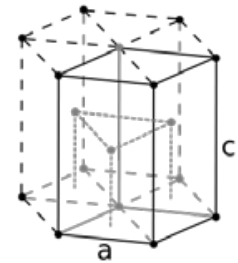

hcp
Figure 7. BCC, FCC and HCP Crystal

\subsection{Orbit Shifting and Phase}

Sc and Ti have hcp structure. As D orbit electron count increases, Mn and Fe become bcc structure by centralize the $\mathrm{D}$ electrons. 
In period 5, the existence of 3D orbit reduces shifting of 4D. Tc and Ru have hcp structure instead.

\subsection{Color of Gold}

Even though there are many theories regarding color of Gold. This paper considers the color of Gold is due to the dissonance of additional electron of $\mathrm{D}$ orbits. It has nine electrons on 3D, 4D and 5D each. Among nine electrons, eight of them are properly paired. Additional electron can not be paired since there are three of them. The active electron interacts with the photon by absorbing blue light to transit the shell from D to $\mathrm{S}$ and creates an unpaired "free" S orbit electron. The newly created unpaired electron does not stay on the S orbit as the orbit is fully occupied. It does not transit back to D orbit as it can not find proper pairing. The photons can be absorbed by these electrons as they are considered to be plasmons, or quasiparticles. These electrons fall into a range of energy band where the visible light can be absorbed and make Gold a distinct color.

This explanation also tells us that silver does not have $\mathrm{D}$ to $\mathrm{S}$ transition, due to the proper $\mathrm{D}$ electron orbit pairing.

\section{Conclusions}

A logical deduction starting from the UFT leads to the understanding of the Crystal structure of elements. The key to solve a structure problem of the materials is deeply rooted in the UFT: from Torque to electron, to Proton, to Cubic atom, finally, to Crystal structure. First two steps are basic theory of the UFT, the next two steps connect UFT to the scientific results known for centuries. It is important to understand the reasons behind the crystal structure of the metals. This paper provides a simple explanation of crystal structure without complex equations. The chemical characteristics of the materials are associated with the movements of electrons and their resonance with the nuclei. Similarly, the electron orbit overlapping and sharing of the neighboring atoms can explain how crystal structures are formed. The paper provides a new tool to study the organism of materials "Physically". The crystal structures of metals largely depend on electron orbits with highest propensities. The angles to the central line of these electrons decide the crystal structure. Among the HCP, FCC and BCC crystals, HCP crystal has largest angle, FCC has second largest angle, BCC has smallest angle.

\section{References}

[1] Cao, Zhiliang, and Henry Gu Cao. "Unified Field Theory and the Configuration of Particles." International Journal of Physics 1.6 (2013): 151-161.

[2] Cao, Zhiliang, and Henry Gu Cao. "Unified Field Theory and Topology of Nuclei." International Journal of Physics 2, no. 1 (2014): 15-22.

[3] Zhiliang Cao, Henry Gu Cao, Wenan Qiang, Unified Field Theory and Topology of Atom, American Journal of Modern Physics. Vol. 4, No. 4, 2015, pp. 1-7.

[4] Zhiliang Cao, Henry Gu Cao. Unified Field Theory. American Journal of Modern Physics. Vol. 2, No. 6, 2013, pp. 292-298.
[5] Cao, Zhiliang, and Henry Gu Cao. "Unified Field Theory and the Hierarchical Universe." International Journal of Physics 1.6 (2013): 162-170.

[6] Cao, Zhiliang, and Henry Gu Cao. "Non-Scattering Photon Electron Interaction." Physics and Materials Chemistry 1, no. 2 (2013): 9-12.

[7] Cao, Zhiliang, and Henry Gu Cao. "SR Equations without Constant One-Way Speed of Light." International Journal of Physics 1.5 (2013): 106-109.

[8] Cao, Henry Gu, and Zhiliang Cao. "Drifting Clock and Lunar Cycle." International Journal of Physics 1.5 (2013): 121-127.

[9] Mehul Malik, Mohammad Mirhosseini, Martin P. J. Lavery, Jonathan Leach, Miles J. Padgett \& + et al. Direct measurement of a 27-dimensional orbital-angular-momentum state vector. Nature Communications, 2014, 5.

[10] H. T. Yuan, M. B. Saeed, K. Morimoto, H. Shimotani, K. Nomura, R. Arita, Ch. Kloc, N. Nagaosa, Y. Tokura, and Y. Iwasa. Zeeman-Type Spin Splitting Controlled with an External Electric Field. Nat. Phys. 2013, 9, 563-569.

[11] A. Rahimi-Iman, C. Schneider, J. Fischer, S. Holzinger, M. Amthor, S. Höfling, S. Reitzenstein, L. Worschech, M. Kamp, and A. Forchel. "Zeeman splitting and diamagnetic shift of spatially confined quantum-well exciton polaritons in an external magnetic field.” Phys. Rev. B 84, 165325-2011, October.

[12] D. Kekez, A. Ljubiic \& B. A. Logan. An upper limit to violations of the Pauli exclusion principle. Nature 348, 224-224 (1990).

[13] Zoran Hadzibabic. Quantum gases: The cold reality of exclusion. Nature Physics 6, 643-644 (2010).

[14] June Kinoshita. Roll Over, Wolfgang? Scientific American 258, 25-28 (1988).

[15] Tony Sudbery. Exclusion principle still intact. Nature 348, 193194 (1990).

[16] R. C. Liu, B. Odom, Y. Yamamoto \& S. Tarucha. Quantum interference in electron collision. Nature 391, 263-265 (1998).

[17] George Gamow. The Exclusion Principle. Scientific American 201, 74-86 (1959).

[18] B. Poirier, Chem. Phys. 370, 4 (2010).

[19] A. Bouda, Int. J. Mod. Phys. A 18, 3347 (2003).

[20] P. Holland, Ann. Phys. 315, 505 (2005).

[21] P. Holland, Proc. R. Soc. London, Ser. A 461, 3659 (2005).

[22] G. Parlant, Y.-C. Ou, K. Park, and B. Poirier, "Classical-like trajectory simulations for accurate computation of quantum reactive scattering probabilities,” Comput. Theor. Chem. (in press)

[23] D. Babyuk and R. E. Wyatt, J. Chem. Phys. 124, 214109 (2006).

[24] Jeremy Schiff and Bill Poirier. Quantum mechanics without wavefunctions. THE JOURNAL OF CHEMICAL PHYSICS 136, 031102 (2012).

[25] J. von Neumann, Mathematical Foundations of Quantum Mechanics (Princeton University Press, Princeton, NJ, 1932).

[26] D. Bohm, Phys. Rev. 85, 166 (1952).

[27] P. R. Holland, The Quantum Theory of Motion (Cambridge University Press, Cambridge, England, 1993).

[28] R. E. Wyatt, Quantum Dynamics with Trajectories: Introduction to Quantum Hydrodynamics (Springer, New York, 2005).

[29] H. Everett III, Rev. Mod. Phys. 29, 454 (1957).

[30] M. F. González, X. Giménez, J. González, and J. M. Bofill, J. Math. Chem. 43, 350 (2008).

[31] Oganessian, Yu. T. et al. (2002). Results from the first 249Cf+48Ca experiment. JINR Communication (JINR, Dubna). http://www.jinr.ru/publish/Preprints/2002/287(D7-2002-287)e.pdf.

[32] Nash, Clinton S. (2005). "Atomic and Molecular Properties of Elements 112, 114, and 118". Journal of Physical Chemistry A 109 (15): 3493-3500.

[33] K.Umemoto, S.Saito, Electronic configurations of superheavy elements, Journal of the physical society of Japan, vol.65, no.10, 1996, p.3175-3179.

[34] Hartmut M. Pilkuhn, Relativistic Quantum Mechanics, Springer Verlag, 2003.

[35] E.Loza, V.Vaschenko. Madelung rule violation statistics and superheavy elements electron shell prediction. http:/arxivweb3.library.cornell.edu/abs/1206.4488

[36] Froese Fischer, Charlotte (1987). "General Hartree-Fock program". Computer Physics Communication 43 (3): 355-365.

[37] Abdulsattar, Mudar A. (2012). "SiGe superlattice nanocrystal infrared and Raman spectra: A density functional theory study". J. Appl. Phys. 111 (4): 044306. 
[38] Hinchliffe, Alan (2000). Modelling Molecular Structures (2nd ed.). Baffins Lane, Chichester, West Sussex PO19 1UD, England: John Wiley \& Sons Ltd. p. 186.

[39] Szabo, A.; Ostlund, N. S. (1996). Modern Quantum Chemistry. Mineola, New York: Dover Publishing.

[40] Levine, Ira N. (1991). Quantum Chemistry (4th ed.). Englewood Cliffs, New Jersey: Prentice Hall. p. 403.

[41] Christophe L. Guillaume, Eugene Gregoryanz, Olga Degtyareva, Malcolm I. McMahon, Michael Hanfland, Shaun Evans, Malcolm Guthrie, Stanislav V. Sinogeikin \& H-K. Mao. Cold melting and solid structures of dense lithium. Nature Physics 7, 211-214 (2011).

[42] Neaton, J. B. \& Ashcroft, N. W. Pairing in dense lithium. Nature 400, 141-144 (1999)

[43] Hanfland, M., Syassen, K., Christensen, N. E. \& Novikov, D. L. New high-pressure phases of lithium. Nature 408, 174-178 (2000).

[44] Shimizu, K., Ishikawa, H., Takao, D., Yagi, T. \& Amaya, K. Superconductivity in compressed lithium at 20?K. Nature 419, 597-599 (2002).

[45] Tamblyn, I., Raty, J. \& Bonev, S. Tetrahedral clustering in molten lithium under pressure. Phys. Rev. Lett. 101, 075703 (2008).

[46] Matsuoka, T. \& Shimizu, K. Direct observation of a pressureinduced metal-to-semiconductor transition in lithium. Nature 458, 186-189 (2009).

[47] Lazicki, A., Fei, Y. \& Hemley, R. High pressure differential thermal analysis measurements of the melting curve of lithium. Solid State Commun. 150, 625-627 (2010).

[48] Hernández, E., Rodriguez-Prieto, A., Bergara, A. \& Alfè, D. Firstprinciples simulations of lithium melting: Stability of the bcc phase close to melting. Phys. Rev. Lett. 104, 185701 (2010).

[49] Datchi, F., Loubeyre, P. \& LeToullec, R. Extended and accurate determination of the melting curves of argon, helium, ice (H2O), and hydrogen (H2). Phys. Rev. B 61, 6535-6546 (2000).

[50] Gregoryanz, E., Goncharov, A. F., Matsuishi, K., Mao, H. K \& Hemley, R. J. Raman spectroscopy of hot dense hydrogen. Phys. Rev. Lett. 90, 175701 (2003).

[51] Börje Johansson, Wei Luo,Sa Li \& Rajeev Ahuja. Cerium; Crystal Structure and Position in The Periodic Table. Scientific Reports 4, Article number: 6398.

[52] Koskenmaki, D. C. \& Gschneidner, K. A., Jr Cerium in: Handbook on the physics and chemistry of rare earths, Vol. 1, eds Gschneidner K. A., \& Eyring L. (Amsterdam, North-Holland), pp 337-377 (1978).

[53] Johansson, B. The a-? transition in cerium is a Mott transition. Philos. Mag. 30, 469-482 (1974).

[54] Gustafson, D. R., McNutt, J. D. \& Roellig, L. O. Positron annihilation in ?- and a-cerium. Phys. Rev. 183, 435-440 (1969).
[55] Kornstädt, U., Lässer, R. \& Lengeler, B. Investigation of the?-a phase transition in cerium by Compton scattering. Phys. Rev. B 21, 1898-1901 (1980).

[56] Rueff, J. P. et al. F-state occupancy at the ?-a phase transition of Ce-Th and Ce-Sc alloys. Phys. Rev. Lett. 93, 067402 (2004).

[57] Loa, I., Isaev, E. I., McMahon, M. I., Kim, D. Y. \& Johansson, B. Lattice dynamics and superconductivity in cerium at high pressure. Phys. Rev. Lett. 108, 045502 (2012).

[58] Lashley, J. C. et al. Tricritical phenomena at the? right arrow a transition in Ce0.9-xLaxTh0.1 alloys. Phys. Rev. Lett. 97, 235701 (2006).

[59] Allen, J. W. \& Martin, R. M. Kondo volume collapse and the ? right arrow a transition in Cerium. Phys. Rev. Lett. 49, 1106-1110 (1982).

[60] Szotek, Z., Temmerman, W. M. \& Winter, H. Self-interaction corrected, local spin density description of the? right arrow a transition in Ce. Phys. Rev. Lett. 72, 1244-1247 (1994).

[61] Peng Tan, Ning Xu \& Lei Xu. Visualizing kinetic pathways of homogeneous nucleation in colloidal crystallization. Nature Physics 10, 73-79 (2014).

[62] Ostwald, W. Studien über die Bildung und Umwandlung fester Körper. 1. Abhandlung: Übersättigung und Überkaltung. Z. Phys. Chem. 22, 289-330 (1897).

[63] Alexander, S. \& McTague, J. Should all crystals be bcc? Landau theory of solidification and crystal nucleation. Phys. Rev. Lett. 41, 702-705 (1978)

[64] Ten Wolde, P. R., Ruiz-Montero, M. J. \& Frenkel, D. Numerical evidence for bcc ordering at the surface of a critical fcc nucleus. Phys. Rev. Lett. 75, 2714-2717 (1995).

[65] Ten Wolde, P. R., Ruiz-Montero, M. J. \& Frenkel, D. Numerical calculation of the rate of crystal nucleation in a Lennard-Jones system at moderate undercooling. J. Chem. Phys. 104, 9932-9947 (1996).

[66] Shen, Y. C. \& Oxtoby, D. W. bcc symmetry in the crystal-melt interface of Lennard-Jones fluids examined through density functional theory. Phys. Rev. Lett. 77, 3585-3588 (1996).

[67] Auer, S. \& Frenkel, D. Crystallization of weakly charged colloidal spheres: A numerical study. J. Phys. Condens. Matter 14, 76677680 (2002).

[68] Moroni, D., ten Wolde, P. R. \& Bolhuis, P. G. Interplay between structure and size in a critical crystal nucleus. Phys. Rev. Lett. 94, 235703 (2005).

[69] Russo, J. \& Tanaka, H. Selection mechanism of polymorphs in the crystal nucleation of the Gaussian core model. Soft Matter 8, 4206-4215 (2012).

[70] Pusey, P. N. \& van Megen, W. Phase behaviour of concentrated suspensions of nearly hard colloidal spheres. Nature 320, 340-342 (1986). 